\title{
The Influence of Socio-Cultural Factors on Perception \& Cognition of Map Symbols
}

\author{
Bradley Denney ${ }^{\mathrm{a}, *}$, Amy L. Griffin ${ }^{\mathrm{b}}$, Zdenêk Stachoň ${ }^{\mathrm{c}}$ \\ ${ }^{a}$ Bespoke Maps \& Data - bradleysdenney@ gmail.com \\ ${ }^{b}$ School of Science, RMIT University - Amy.Griffin@ rmit.edu.au \\ ${ }^{c}$ Department of Geography, Masaryk University - zstachon@geogr.muni.cz \\ * Corresponding author
}

Keywords: analytic vs. holistic, cartographic perception, cognitive styles, cultural differences, map-reading

\begin{abstract}
:
This experiment was part of a larger study of cultural differences in cartographic cognition led by Masaryk University in the Czech Republic. This study collected data relating to Australians and compared them to previously collected data from Chinese participants. The research sought to investigate how cultural factors and writing system affect the perception of cartographic symbols. Specifically, it built Stachon et al. (2019) that compared Chinese and Czech participants, to gain further insight into cultural differences in cartographic perception. The research attempted to address the following questions:
\end{abstract}

1. How do Australian and Chinese users' perception of point symbols compare to each other?

2. What are the differences in how map users who write with an alphabetic or logographic writing system perceive cartographic point symbols?

3. What role does analytic vs. holistic thinking have in cartographic symbol interpretation?

Miyamoto (2013) suggested that a cultural difference in cartographic perception would be observed, with Australians performing better in tasks requiring a local evaluation of the symbols and Chinese participants preferring global thinking processes.

The full value of geospatial analysis is only reached if it can be communicated with other people, and this is often done through maps. It is also known that the most effective communication requires an understanding of the audience and making it as easy as possible to interpret the information being conveyed. Miyamoto (2013) had shown a demonstrable difference in cognitive processing patterns between those from Eastern and Western cultures. Therefore, this research investigated this phenomenon and its effect on map-reading behaviours among those from Australian and Chinese cultural groups.

This study aimed to investigate cultural differences in cartographic perception through empirical research with map users. In this study, participants from two different cultures, Australian and Chinese, completed cognitive and map reading tasks. Due to the COVID-19 pandemic, Australians were recruited online using Prolific and completed this experiment remotely, while the Chinese experiment was conducted on-campus before the pandemic began at Nanjing Normal University, China. For both groups, participants completed a compound figures test and visual search task on Hypothesis, a platform developed for cartographic research experiments in Šašinka, Morong and Stachoň (2017).

The compound figures test (Figure 1, left) was chosen for its ability to test both analytic against holistic cognitive styles, and global against local cognitive processing strategies with standard small and large figures. The visual search task tested participants' abilities to locate symbols on tourist maps, using two different symbol sets, iconic and schematic (Figure 1, right). The results of these samples were then cleaned, with outliers removed, and analysed using a combination of Detrended Normal Q-Q Plots, Shapiro-Wilk Test of Normality, and mixed and two-way repeatedmeasures analyses of variance. The null hypothesis was that there was no difference in cartographic perception and cognition between map readers from the two cultures. As this study collected and used data from human participants, its procedure was evaluated by the relevant ethics advisory body, which approved the experiment, noting that it followed an ethical procedure and presented a low risk to participants. 


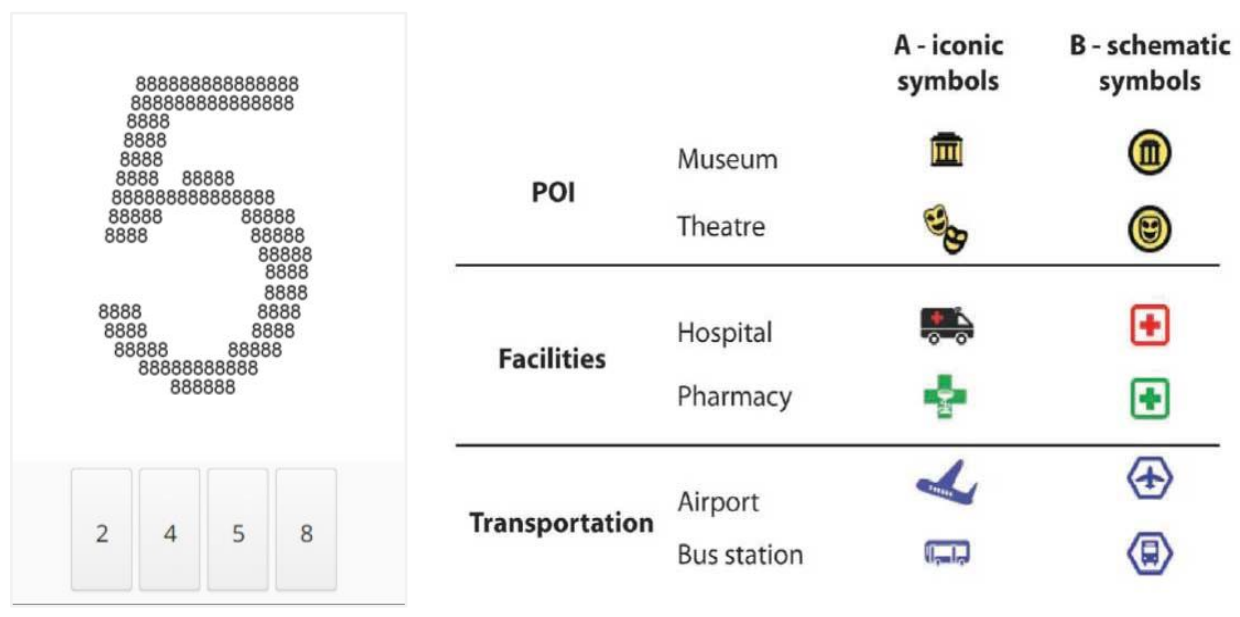

Figure 1. Compound figures test (left) and visual search experimental stimuli (right)

The results of the compound figures test indicated that both Australian and Chinese groups had similar levels of preference towards global cognitive processing, which was not expected given existing research into cultural differences but was consistent with Lacko et al. (2020), which investigated the difference between Czech and Chinese participants. For the visual search task, both Australian and Chinese participants performed better with the iconic symbol set, with the Chinese participants being more heavily affected by the symbol set used. This was unexpected, as a cultural difference in symbol preferences was not only suggested by past research into the underlying theories but also seen in Stachoň et al. (2019) using a similar map-reading task. While differences in perception related to writing systems may exist, our results suggest that these differences do not propagate into cartographic perception or are too small to be noticed by the design of this experiment. Contrary to expectations, a clear link between analytic vs. holistic cognitive styles was not found in this experiment. This may be, in part, due to the nature of the experimental task or perhaps due to the highly educated background of the participants in both groups.

These results suggest in agreement with Stachoň et al. (2020), that while there were few cultural differences observed, the value of well-established cartographic principles, such as visual contrast, may be even more important when creating maps for multicultural audiences. The role of cognitive styles in map reading requires further investigation, and this study has helped give more context to both Australian and Chinese map users.

Further research is required to better understand the role of socio-cultural factors in cartographic perception. Future directions of research into this field could aim to investigate a wider set of cultures or differences in reading other map types. Additionally, further analysis of this study's research data may provide more insight into other demographic factors affecting the perception and cognition of maps. In both cases, future research will provide more information and clarity on the issue of cultural differences in cognitive processes used in map reading.

\section{Acknowledgements:}

We would like to thank Professor Jie Shen of Nanjing Normal University, China for her support in facilitating the Chinese experiment and recruiting Chinese participants and the project team of the Czech Science Foundation grant "The Influence of Socio-Cultural Factors and Writing Systems on the Perception and Cognition of Complex Visual Stimuli" (GC19-09265J) for providing the infrastructure.

\section{References}

Lacko D, Šašinka Č, Čeněk J, Stachoň Z and Lu WL (2020) 'Cross-Cultural Differences in Cognitive Style, Individualism/Collectivism and Map Reading between Central European and East Asian University Students', Studia Psychologica, 62(1):23-43, doi:10.31577/sp.2020.01.789.

Miyamoto Y (2013) 'Culture and Analytic Versus Holistic Cognition: Toward Multilevel Analyses of Cultural Influences', in Devine P and Plant A (eds) Advances in Social Psychology, Academic Press, 47:131-188, doi:10.1016/B978-0-12-407236-7.00003-6.

Šašinka Č, Morong K and Stachoň Z (2017) 'The Hypothesis Platform: An Online Tool for Experimental Research into Work with Maps and Behavior in Electronic Environments', ISPRS International Journal of Geo-Information, 6(12):407-428, doi:10.3390/ijgi6120407.

Stachoň Z, Šašinka Č, Čeněk J, Štěrba Z, Angsuesser S, Fabrikant SI, Štampach R and Morong K (2019) 'Crosscultural differences in figure-ground perception of cartographic stimuli', Cartography and Geographic Information Science, 46(1):82-94, doi:10.1080/15230406.2018.1470575.

Stachoň Z, Šašinka Č, Konečný M, Popelka S and Lacko D (2020) 'An Eye-Tracking Analysis Of Visual Search Task On Cartographic Stimuli', in Bandrova T, Konečný M and Marinova S (eds) 8th International Conference on Cartography \& GIS: Proceedings, Bulgarian Cartographic Association, 1:36-41. 